\title{
CACERÍA DE SUBSISTENCIA EN SAN MARCOS, SUCRE, COLOMBIA
}

\section{SUBSISTENCE HUNT IN SAN MARCOS, SUCRE, COLOMBIA}

\author{
DE LA OSSA, V. JAIME ${ }^{1^{*}}$ Dr., DE LA OSSA-LACAYO, ALEJANDRO ${ }^{2}$ Ecólogo. \\ ${ }^{1}$ Universidad de Sucre, Facultad de Ciencias Agropecuarias, Grupo de Investigación \\ en Biodiversidad Tropical, Colombia. ${ }^{2}$ Pontificia Universidad Javeriana, Maestría en \\ Desarrollo Rural, Colombia.
}

*Correspondencia: jaimedelaossa@yahoo.com

Recibido: 12-05-2011; Aprobado: 22-08-2011

\section{Resumen}

Este estudio analiza la caza de subsistencia tomando como modelo las especies de reptiles, aves y mamíferos que son capturados para consumo o comercio en la zona de San Marcos, Sucre, Colombia. La zona referida se caracteriza por la presencia de extensas ciénagas y formar parte de la región de la Mojana. Se aplicaron entrevistas semiestructuradas a habitantes rurales, conocedores y usuarios del recurso. Se evidencia que las especies de mayor preferencia, caracterizadas por un mayor valor de uso, son aquellas que de forma particular están asociadas a la tradición de consumo cultural. Los registros permiten conocer de preliminarmente el estado de la fauna silvestre que se está utilizando para proponer futuros estudios ambientales que conduzcan a proyectos de conservación.

Palabras clave: fauna silvestre, utilización, San Marcos, Sucre, Colombia.

\section{Abstract}

The subsistence hunt taking as model the species of reptiles, birds and mammals that are captured for consumption or trade in San Marcos, Sucre, Colombia, was analyzed The referred area is characterized by the presence of extensive marshes and it is part of the region of the Mojana. Semistructured interviews were applied to the rural inhabitants, experts and users of the resource. It is evidenced that the species of more preference, are characterized by a bigger use value, are those that are associated to the tradition of cultural consumption in a particular way. In essence the registrations allow introductorily knowing the state of the wildlife that is using to propose future environmental studies that lead to conservation projects.

Key words: wildlife, use, San Marcos, Sucre, Colombia.

\section{Introducción}

Los animales silvestres han sido de primordial importancia para la especie humana al ser utilizados como fuente de alimentación y para la elaboración de diversos utensilios (REDFORD y ROBINSON, 1991; OJASTI, 2000; ROBINSON y BENNETT, 2000). Todavía hacen parte de la mitología y el arte 
en muchas culturas (STEARMAN, 2000), mientras que algunos son utilizados como animales de compañía o para exhibición (REDFORD y ROBINSON, 1991).

La utilización de la fauna silvestre es muy antigua, se sitúa en los orígenes mismos del hombre, cuando era sin duda parte importante de su dieta y comercio, y marcó gran influencia en el desarrollo cultural de la humanidad (DE LA OSSA, 1998; BAPTISTE-BALLERA et al., 2002). La importancia económica de la fauna no es fácilmente evaluable a nivel de su comercio internacionalmente. Regional y localmente es aún más difícil, entran en juego las diferentes características de los grupos humanos, la disponibilidad relativa ante otros recursos y el contexto sociopolítico y simbólico regional (BAPTISTEBALLERA et al., 2002), además de los aspectos legales y normativos sobre uso y la paradoja entre cultura, necesidad de subsistencia y paradigmas de la conservación, que se entrelazan en una mezcla que favorece el consumo ilegal y propician la ilegalidad (OJASTI, 2000).

Entre las especies de fauna silvestre de mayor importancia comercial y de consumo en Colombia, están: Hydrochaeris hydrochaeris (ponche, chigüiro), Agouti paca (guartinaja, boruga, paca), Tayassu sp. (zaino, puerco de monte), Dasyprocta sp (ñeque, picure), Odocoileus virginianus (venado), Mazama sp (venado soche), Dasypus novencinctus (armadillo), Silvilagus floridanus (conejo), Iguana iguana (iguana), Trachemys callirostris (hicotea) (PÉREZ y OJASTI, 1996; RAMÍREZ-PERILLA, 1996; AGUILERA-DÍAZ, 2004). En general, el consumo de carne de animales silvestres resulta ser el uso más importante dado a este componente en todo país, incluso en algunas regiones en donde existe la más alta oferta de ganado vacuno (POLANCO, 2000).

El municipio de San Marcos, Sucre, por su ubicación geográfica y por razones de índole cultural ha sido centro de explotación de fauna silvestre, con raíces sociales y culturales muy profundas (DE LA OSSA, 2003; AGUILERA-DÍAZ, 2004). El conocimiento que tienen los pescadores o cazadores locales, permite abordar con mayor objetividad los procesos de aprovechamiento de la fauna silvestre en un contexto consuntivo, que puede servir como base para aplicar políticas integrales de conservación sin desconocer la realidad social, económica y cultural circundante (ALDANA et al., 2006).

Se busca con este trabajo estimar la importancia de los reptiles, aves y mamíferos silvestres reconocidos y utilizados por la comunidad rural del municipio de San Marcos en el departamento de Sucre; se propone con base en los resultados obtenidos por entrevista, la representatividad de cada taxón en este proceso de aprovechamiento. 


\section{Materiales y métodos}

Caracterización del área de estudio: el municipio de San Marcos (Fig. 1) está situado en el suroeste del departamento de Sucre a $8^{\circ} 38^{\prime} \mathrm{N}$ y $75^{\circ} 04^{\prime} \mathrm{O}$, entre 15 y 20 m.s.n.m, en la denominada Región de la Mojana, depresión Momposina, Colombia. Este sistema ambiental actúa como regulador de los ríos Magdalena, Cauca y San Jorge; mayoritariamente la zona está conformada por humedales (AGUILERA-DÍAZ, 2004); además de poseer importantes y variados hábitat para la fauna silvestre regional y ser por excelencia un lugar de tradicional extractivismo faunístico (DE LA OSSA, 2003). La zona está conformada por llanuras fluvio deltaicas y fluvio lacustres; con zonas de bosque húmedo tropical, bosque seco tropical, bosque muy seco tropical y sabanas naturales (AGUILERA-DÍAZ, 2005).

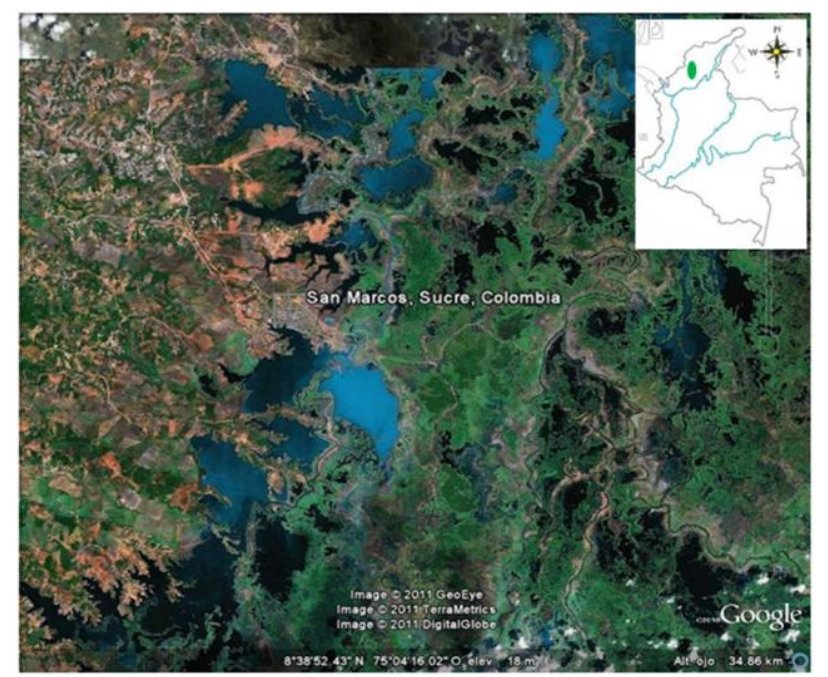

Figura 1. Área de estudio (Google Earth, versión 5.0)

Muestreos: para la identificación de los usos de la fauna se realizaron en la zona rural de San Marcos, Sucre, entre abril de 2008 y marzo de 2009, un total de cuatro sesiones de trabajo, equivalentes a una sesión cada tres meses. Se aplicaron un total de 134 entrevistas semiestructuradas a pescadores y/o extractivistas previamente identificados, procedentes de la zona inundable, con permanencia en la zona de por lo menos 20 años (MARGOLUIS y SALAFSKY, 1998); la muestra cubrió el $60 \%$ de los extractivistas activos. Se llevó a cabo una comprobación de imágenes para reconocimiento de cada especie registrada en las encuestas por parte del entrevistado (HILTY y BROWN, 1986; EMMONS, 1991; RENGIFO y LUNDBERG, 1999).

Análisis de la información: Los usos se clasificaron en siete categorías: carne (consumo o venta) (C/C/V), mascotas (tenencia en casa) (M/C), huevos 
(consumo o venta) $(\mathrm{H} / \mathrm{C} / \mathrm{V})$, crías (venta) $(\mathrm{C} / \mathrm{V})$, piel (venta) $(\mathrm{P} / \mathrm{V})$, medicinal $(\mathrm{M})$ y daños (sembrados o producción pecuaria) (D).

\section{Resultados}

En total se registró por entrevista la utilización de un total de 48 especies (Tabla 1) de las cuales se tienen siete especies de reptiles (14,6\%), 30 especies de aves (62,5\%) y 11 especies de mamíferos (22,9\%). En la misma Tabla se incluye la relación de los diferentes usos dados a cada una de las especies halladas en este trabajo. En cuanto a los usos dado por especie, se tiene: C/C/V 27 (56,2\%), M/C 18 (37,5\%), H/C/V 7 (14,6\%), C/V 22 (45,8\%) P/V $3(6,25 \%)$, M $15(31,2 \%)$, D $15(31,2 \%)$.

Tabla 1. Uso de reptiles, aves y mamíferos detectados en la zona de estudio

\begin{tabular}{|c|c|c|c|c|c|c|c|c|c|c|}
\hline Especie & N. Común & Taxón & $\mathrm{C} / \mathrm{C} / \mathrm{V}$ & $\mathrm{M} / \mathrm{C}$ & $\mathrm{H} / \mathrm{C} / \mathrm{V}$ & $\mathrm{C} / \mathrm{V}$ & $\mathbf{P} / \mathbf{V}$ & $\mathbf{M}$ & D & To \\
\hline Iguana iguana & Iguana & $\mathrm{R}$ & 1 & 1 & 1 & 1 & & 1 & & 5 \\
\hline Tupinambis teguixin & Lobo pollero & $\mathrm{R}$ & & & & 1 & & 1 & & 2 \\
\hline Boa constrictor & boa & $\mathrm{R}$ & & 1 & & 1 & 1 & 1 & & 4 \\
\hline Trachemys callirostris & Hicotea & $\mathrm{R}$ & 1 & 1 & 1 & 1 & & 1 & & 5 \\
\hline Rhinoclemmys melanosterna & Galápago & $\mathrm{R}$ & 1 & 1 & & & & & & 2 \\
\hline Podocnemis lewyana & Tortuga & $\mathrm{R}$ & 1 & 1 & 1 & 1 & & 1 & & 5 \\
\hline Caiman crocodylus fuscus & Babilla & $\mathrm{R}$ & 1 & & 1 & 1 & 1 & 1 & 1 & 6 \\
\hline Phalacrocorax olivaceus & Pato yuyo & A & 1 & & & & & & & 1 \\
\hline Anhinga anhinga & Pato aguja & $\mathrm{A}$ & 1 & & & & & & & 1 \\
\hline Ardea herodias & Garza & $\mathrm{A}$ & 1 & & & & & & & 1 \\
\hline Ardea cocoi & Garza morena & $\mathrm{A}$ & 1 & & & & & & & 1 \\
\hline Mycteria americana & Coyongo & $\mathrm{A}$ & 1 & & & & & & & 1 \\
\hline Chauna chavaria & Chavarrí & $\mathrm{A}$ & 1 & 1 & & & & & & 2 \\
\hline Dendrocyna viduata & Viudita & A & 1 & & 1 & 1 & & & & 3 \\
\hline Dendrocygna autumnalis & Pisingo & A & 1 & & 1 & 1 & & & & 3 \\
\hline Cairina moschata & Pato real & A & 1 & & 1 & 1 & & & & 3 \\
\hline Pandion haliatus & Águila pescadora & $\mathrm{A}$ & & & & & & & 1 & 1 \\
\hline Busarellus nigricollis & Bebe humo & $\mathrm{A}$ & & & & & & & 1 & 1 \\
\hline Buteo magnirostris & Gavilán pollero & $\mathrm{A}$ & & & & & & & 1 & 1 \\
\hline Milvago chimachima & Pigua & $\mathrm{A}$ & & & & & & & 1 & 1 \\
\hline Ortalis garrula & Guacharaca & A & 1 & & & 1 & & & & 2 \\
\hline Penelope purpurascens & Pavita & A & 1 & & & 1 & & & & 2 \\
\hline Colinus cristatus & Perdíz & $\mathrm{A}$ & 1 & & & & & & & 1 \\
\hline Burhinus bistriatus & Galán & $\mathrm{A}$ & & 1 & & & & & & 1 \\
\hline Columba cayennensis & Guarumera & $\mathrm{A}$ & 1 & & & 1 & & 1 & & 3 \\
\hline Columbina minuta & Tierrerita & A & 1 & & & & & & & 1 \\
\hline Columbina talpacoti & Tortolita & A & 1 & & & & & & & 1 \\
\hline
\end{tabular}




\begin{tabular}{|c|c|c|c|c|c|c|c|c|c|}
\hline Leptotila verreauxi & Caminera & A & 1 & & & & & & 1 \\
\hline Ara ararauna & Gonzalo & A & & 1 & 1 & & & & 2 \\
\hline Aratina pertinax & Cotorra & A & & 1 & 1 & & & 1 & 3 \\
\hline Brotogeris jugularis & Perico & $\mathrm{A}$ & & 1 & 1 & & & 1 & 3 \\
\hline Amazona ochrocephala & Loro & $\mathrm{A}$ & & 1 & 1 & & & 1 & 3 \\
\hline Ramphastus sulfuratus & Guazalé & A & & 1 & & & & & 1 \\
\hline Thraupis episcopus & Azulejo & $\mathrm{A}$ & & 1 & & & & 1 & 2 \\
\hline Volantina jacarina & Mochuelo & A & & 1 & & & & & 1 \\
\hline Sphorophilla minuta & Meriño & A & & 1 & & & & & 1 \\
\hline Sicalis flaveola & Canario & $\mathrm{A}$ & & 1 & & & & & 1 \\
\hline Didelphis marsupialis & Zorra chucha & $\mathrm{M}$ & & & & & 1 & 1 & 2 \\
\hline Dsypus novemcinctus & Armadillo & $\mathrm{M}$ & 1 & & & & 1 & & 2 \\
\hline Alouatta seniculus & Mono & $\mathrm{M}$ & & 1 & 1 & & 1 & & 3 \\
\hline Aotus lemurinus & Marta & $\mathrm{M}$ & & 1 & & & & & 1 \\
\hline Cerdocyon thous & Zorra perro & M & & & & & & 1 & 1 \\
\hline Leopardus tigrina & Gato de monte & $\mathrm{M}$ & & & & & & 1 & 1 \\
\hline Tayassu tajacu & Zaino & $\mathrm{M}$ & 1 & & 1 & & 1 & & 3 \\
\hline Mazama americana & Venado & $\mathrm{M}$ & 1 & & 1 & 1 & 1 & & 4 \\
\hline Hydrocaheris hydrochaeris & Ponche & $\mathrm{M}$ & 1 & & 1 & & 1 & 1 & 4 \\
\hline Agouti paca & Guartinaja & $M$ & 1 & & 1 & & 1 & 1 & 4 \\
\hline Dasyprocta punctata & Ñeque & $\mathrm{M}$ & 1 & & 1 & & 1 & 1 & 4 \\
\hline
\end{tabular}

$\mathrm{R}=$ Reptil, $\mathrm{A}=$ Ave, $\mathrm{M}=$ Mamífero, To = Total, $\mathrm{C} / \mathrm{C} / \mathrm{V}=$ Carne (consumo o venta), $\mathrm{M} / \mathrm{C}=$ mascotas (tenencia en casa), $\mathrm{H} / \mathrm{C} / \mathrm{V}=$ huevos (consumo o venta), $\mathrm{C} / \mathrm{V}=$ crías (venta), $\mathrm{P} / \mathrm{V}=$ piel (venta), $\mathrm{M}$ $=$ medicinal, $\mathrm{D}=$ daños (sembrados o producción pecuaria).

En la Tabla 2, se presenta información del uso de acuerdo con el taxón analizado, es evidente significativamente que el consumo de carne de fauna silvestre se presenta con mayor énfasis en aves, mientras que para reptiles y mamíferos se aproxima porcentualmente. Se tiene que el uso de mascotas preferencialmente se dirige a las aves; mientras que el consumo de huevos se ubica con mayor presión sobre reptiles al igual que la venta de pieles; es evidente que lo relacionado con medicina tradicional vincula en su orden a mamíferos y reptiles preferencialmente.

Tabla 2. Comportamiento porcentual de los usos detectados de acuerdo con el taxón

\begin{tabular}{|l|c|c|c|c|c|c|c|}
\hline Taxón & $\mathbf{C / C} / \mathbf{V}$ & $\mathbf{M} / \mathbf{C}$ & $\mathbf{H} / \mathbf{C} / \mathbf{V}$ & $\mathbf{C} / \mathbf{V}$ & $\mathbf{P} / \mathbf{V}$ & $\mathbf{M}$ & $\mathbf{D}$ \\
\hline Reptiles & $5(17,24 \%)$ & $5(17,24 \%)$ & $4(13,79 \%)$ & $6(20,7 \%)$ & $2(6,89 \%)$ & $6(20,7)$ & $1(3,44 \%)$ \\
\hline Aves & $16(32,5 \%)$ & $11(22,4 \%)$ & $3(6,1 \%)$ & $10(20,4 \%)$ & 0 & $1(2,3 \%)$ & $8(16,3 \%)$ \\
\hline Mamíferos & $6(20,7 \%)$ & $2(6,9 \%)$ & 0 & $6(20,7 \%)$ & $1(3,4 \%)$ & $8(27,6 \%)$ & $6(20,7 \%)$ \\
\hline
\end{tabular}




\section{Discusión}

Los procesos de desarrollo socioeconómico de la región, se separan ampliamente del bienestar general de la comunidad, si bien existe acceso a muchos bienes de consumo y en especial a otros ítems alimentares, el ingreso per capita es bajo y la marginalidad o aislamiento socioeconómico persisten en una significativa porción de la población (DE LA OSSA, 2003; AGUILERADÍAZ, 2004).

El extractivismo de fauna silvestre se relaciona con la facilidad de acceso a productos alimenticios comerciales, la fauna silvestre cobra mayor importancia en la dieta, llegando a cubrir un elevado porcentaje de los requerimientos de proteína en grupos indígenas, colonos y comunidades marginales urbanas y rurales, como lo señala OJASTI (1993).

En la explotación de la fauna silvestre, además del aspecto cultural, la facilidad de acceso a la captura durante algunas épocas del año, la ausencia de producción pecuaria alternativa y doméstica menor, el bajo ingreso económico y la marginalidad, hacen que la cacería sea una alternativa viable de subsistencia (REDFORD y ROBINSON, 1991; ALVARD, 2000; HILL y PADWE, 2000; DE LA OSSA, 2003; FUENTES-OBEID et al., 2003).

La utilización de la fauna silvestre se registra para toda América latina como de vital importancia social, es así como muchas especies de reptiles, aves y mamíferos mantienen una estrecha relación con la economía de estos grupos humanos, parte de la producción es destinada al consumo y otra parte se usa como bien de cambio (OJASTI, 1993; AQUINO et al., 2001; PERES, 2001; MONROY-VILCHIS et al., 2008), como se muestra en los usos dados por especie, en donde coexisten ambas modalidades, siendo la carne el valor sobresaliente con $56,2 \%$.

Los resultados muestran que no existe una preferencia general por un taxón dado, lo cual puede estar relacionado con: la talla, esfuerzo de captura, disponibilidad temporal y arraigo en los factores culturales de uso. En general, La presión de caza varía dependiendo de la especie, en ecosistemas intactos o preservados los grandes mamíferos están entre los más apreciados, le siguen en su orden aves y reptiles (VICKERS, 1984; REDFORD y ROBINSON, 1991).

Se evidencia en este estudio que la cacería de aves para alimentación y mascotas ocupa el primer lugar, mientras que para utilización de huevos y pieles los más altos índices de uso se ubican en los reptiles, siendo $C$. crocodilus fuscus, I. iguana y los quelonios los de mayor representatividad, son 
utilizados tanto para alimentación como para el comercio de pieles (DE LA OSSA, 2003). Las aves son relativamente de mayor importancia en la dieta (ALVARD et al., 1997), se tiene un mayor número de especies y su consumo es significativo.

La utilización generalizada y preferencial de $T$. callirostris (hicotea) hace que la región y su área de influencia sea particularmente característica para este tipo de extracción dentro del territorio nacional, como ya se ha registrado (DE LA OSSA, 2003; FUENTES-OBEID et al., 2003), al igual que lo es para l. iguana (DE LA OSSA, 2003), cuyo consumo preferencial y significativamente mayoritario son los huevos. En América Central y Suramérica, la iglesia católica apoyó el uso de Trachemys spp, durante las celebraciones de cuaresma y semana santa, tradición que aún persiste (PRITCHARD y TREBBAU, 1984; GÓMEZ et al., 2008) y que como se observa no es ajena a la zona de estudio y que se ha extendido a otras especies, como: $H$. hydrochaeris e I. iguana, con similitud de consideración cultural católica.

El caso de los quelonios y de $C$. crocodilus fuscus es comparable con el proceso de extracción que se tiene para algunas áreas latinoamericanas de amplios humedales, como la cuenca amazónica, por ejemplo, en donde la oportunidad de acceso y la masiva presencia los hacen presa fácil para su captura y porque culturalmente poseen una amplia y antigua tradición de uso (REDFORD y ROBINSON, 1991; OJASTI, 1993; RODRÍGUEZ y ROJASSUAREZ, 1995), como sucede en el municipio de San Marcos, en donde además el comercio ilegal ejerce fuerte influencia sobre el extractivismo de estos reptiles todo el año.

Existe un mercado creciente que se mueve alrededor de la compra de neonatos y vincula en este estudio principalmente a aves y reptiles de forma representativa, situación que podría impactar mucho más la estabilidad de las ecosistémica, ya que se estaría eliminando la posibilidad de renovación poblacional. Este tipo de extracción, la mayoría de las veces orientada hacia el contrabando de especies silvestres como mascotas es una modalidad de aprovechamiento comercial ilegal altamente nociva (REDFORD y ROBINSON, 1991; OJASTI, 1993; BAPTISTE-BALLERA et al., 2002; DE LA OSSA, 2003).

La cacería de mascotas y la venta de subproductos derivados, en este caso puede enmarcarse dentro de patrones sociales de subsistencia, aún cuando legalmente la cacería de subsistencia esté referida tácitamente a la alimentación del usuario, según lo preceptuado en el Decreto 1608/1978, Art. 31, señala: "El aprovechamiento de la fauna silvestre y de sus productos sólo podrá adelantarse mediante permiso, autorización o licencia que se podrán obtener en la forma prevista por este Decreto". Agrega de forma puntual y única: "La caza de 
subsistencia no requiere permiso pero deberá practicarse en forma tal, que no se causen deterioros al recurso. La entidad administradora organizará sistemas para supervisar su ejercicio". El sistema acusa una moratoria de 23 años.

Los resultados obtenidos indican que los pobladores rurales de este estudio, cazan con fines de subsistencia, la alternativa comercial les ofrece la posibilidad de obtener ingresos adicionales que les ayudan a sufragar gastos que también aportan a su diario vivir, aunque normativamente actúen en la ilegalidad. En algunos casos la cacería se orienta a la eliminación selectiva de algunas especies que consideran perjudiciales (REBÊLO y PEZZUTI, 2001), lo cual también hace parte del universo cultural en el que habitan, queda demostrado especialmente en aves y mamíferos que afectan sus cultivos, como lo muestran los resultados.

La medicina tradicional posee como característica básica una fuerte y necesaria vinculación entre lo cultural individual y lo social (MOLINA y PEÑALOZA, 2002), específicamente la fauna silvestre también posee vinculación con la medicina tradicional y aunque su uso es menor que el de las plantas, está presente en las comunidades nativas como componente importante (TORRES, 1999; BARBARÁN 2004). Así lo muestran los resultados al referenciar la utilización de ciertas especies con fines curativos, que expresan el uso para este fin del $31,2 \%$ de las especies totales detectadas, en donde mamíferos y reptiles son los de mayor utilización. Se trata de un valor social importante para la comunidad, comparable con resultados similares a los hallados en México (CORTEZ et al., 2004; ENRÍQUEZ-VÁZQUEZ et al., 2006). Aspecto que no ha sido tenido en cuenta particularmente por la normatividad colombiana, que está poco estudiado en nuestro territorio y que requiere ser abordado científicamente.

Los efectos de la cacería sobre la fauna silvestre así como sobre los ecosistemas han sido evaluados en diversos estudios, los cuáles muestran como se altera la composición faunística y florística, a veces con consecuencias drásticas para la diversidad biológica y la subsistencia humana (PERES, 2000), llegando a ser tan graves los efectos en la mayoría de los bosques tropicales que los vertebrados de mayor tamaño desaparecen rápidamente en hábitat relativamente intactos, y esto puede ocasionar el "efecto de bosque vacío" (ROBINSON y BENNETT, 2000); también estos efectos en ciertos casos podrían relacionarse con la alteración de la sinergia existente en el proceso <<fuente-sumidero>> (ROBINSON y BENNETT, 2000; PERES, 2001), lo que en este caso podría estar afectando las poblaciones de mamíferos y reptiles, trasladando la captura mayoritaria hacia las aves. 


\section{Referencias}

AGUILERA-DÍAZ, M. 2004. La Mojana: riqueza natural y potencial económico. Banco de la República. Serie de documentos de trabajo sobre economía regional $N^{\circ}$. 48. Cartagena, Colombia.

AGUILERA-DÍAZ, M. 2005. La economía del departamento de Sucre: ganadería y sector público. Banco de la República. Serie de Documentos de trabajo sobre economía regional $N^{\circ} 63$. Cartagena, Colombia.

ALDANA, N.J.; DÍAZ-PORRES, M.; FEIJOO, M.A.; ZÚÑIGA, M.C. 2006. Valoración del uso de la fauna silvestre en el municipio de Alcalá, Valle del Cauca. Scientia et Technica XII (31):291-296.

ALVARD, M.; ROBINSON, J.; REDFORD, K.; KAPLAN, H. 1997. The sustainability of subsistence hunting in the Neotropics. Conservation Biology 4:977-982.

ALVARD, M. 2000. The impact of traditional subsistence hunting and trapping populations: data from Wana horticulturalists of Upland Central Sulawesi, Indonesia. Págs. 214-230. En: Robinson, J.G; Redford, K.H. (Eds.). Neotropical Wildlife Use and Conservation. Chicago University Press, Chicago, USA.

AQUINO, R.; PACHECO, T.; VÁSQUEZ, M. 2001.Evaluación y valorización económica de la fauna silvestre en el río Algodón, Amazonía peruana. Rev. peru. biol. 14(2):187-192.

BAPTISTE-BALLERA, L.G.; HERNÁNDEZ-PÉREZ, S.; POLANCO-OCHOA, R.; QUICENO-MESA, M.P. 2002. La fauna silvestre colombiana: una historia económica y social de un proceso de marginalización. Disponible en: http://www.humboldt.org.co/pdf/usoyval/Baptiste.pdf (Consultado: 15-07-2010).

BARBARÁN, F.R. 2004. Usos mágicos, medicinales y rituales de la fauna en la Puna del Noroeste Argentino y sur de Bolivia. Contribuciones al manejo de vida silvestre en Latinoamérica 1:1-26.

CORTEZ, V.; MACEDO, J.; HERNÁNDEZ, M.; ARTEAGA, G.; ESPINOSA, D.; RODRÍGUEZ, J. 2004. Farmacognosia: breve historia de sus orígenes y su relación con las ciencias médicas. Revista Biomédica 15:123-136.

DE LA OSSA, V.J. 1998. Talleres académicos introductorios para la cría y manejo de algunos reptiles. Universidad de Sucre, Facultad de Ciencias Agropecuarias. Sincelejo, Colombia. 
DE LA OSSA, V.J. 2003. Manejo de fauna silvestre tropical. Programa de Desarrollo Sostenible de la Región de La Mojana. DNP, FAO, Bogotá, Colombia.

EMMONS, L.H. 1991. Neotropical Rainforest Mammals. A Field Guide. The University of Chicago Press. Chicago, USA.

ENRÍQUEZ-VÁZQUEZ, P.; MARIACA-MÉNDEZ, R.; RETANA-GUIASCÓN, O.G.; NARANJO-PIÑERA, E.J. 2006. Uso medicinal de la fauna silvestre en los altos de Chiapas, México. Interciencia 31(7):491-499.

FUENTES-OBEID, S.; SAMPEDRO, M.A.; ARDILA-MARULANDA, M. 2003. Importancia de la jicotea (Trachemys scripta callirostris: Chelonia, Emydidae) como recurso natural en la comunidad de isla del Coco, Región de La Mojana, Departamento de Sucre, Colombia. Revista Biología 17(2):126-133.

GÓMEZ, A.H.; MOLINA, M.; CASTRO, M.; TELLERIA, B.M. 2008. Factores socioecológicos que amenazan a la vida silvestre en la vertiente sur del Parque Nacional Sierra Nevada, Venezuela. Producción Agropecuaria 1(1):40-49.

HILL, K.; PADWE, J. 2000. Sustainability of Aché Hunting in the Mbaracayú Reserve, Paraguay. Págs. 79-105. En: Robinson, J.G.; Bennett, E.L. (Eds.). Hunting for Sustainability in Tropical Forests. Columbia University, New York, USA.

HILTY, S.; BROWN, W. 1986. A Guide of the Birds of Colombia. Princeton University Press. Princeton, USA.

MARGOLUIS, R.; SALAFSKY, N. 1998. Measures of success: designing, managing, and monitoring conservation and development projects. Island Press. Washington, DC, USA..

MOLINA, M.; PEÑALOZA, J. 2002. Dog predation on paramo white-tailed deer: the case Mucubaji, Sierra Nevada National Park. Memoria de la Fundación la Salle de Ciencias Naturales 154:139-144.

MONROY-VILCHIS, O.; CABRERA, L.; SUAREZ, P.; ZARCO-GONZÁLEZ, M.M.; RODRÍGUEZ-SOTO, C.; URIOS, V. 2008. Uso tradicional de vertebrados silvestres en la Sierra Nacghititla, México. INCI 33(4):308-313.

OJASTI, J. 1993. Utilización de la fauna silvestre en América Latina, situación y perspectiva para un manejo sostenible. Guía FAO, Conservación № 25, Roma; 
OJASTI, J. 2000. Manejo de Fauna Silvestre Neotropical. SIMAB Series No 5. Smithsonian Institution / MAB Program. Washington, D. C, USA.

PÉRES, C.A. 2000. Effects os subsistence hunting on vertebrate community structure in Amazonian forests. Conservation Biology 14 (1):240-253.

PÉRES, C.A. 2001. Synergistic effect of subsistence hunting habitat fragmentation on Amazonian forest vertebrates Conservation Biology 15(6):1490-1505.

PÉREZ, E.M.; OJASTI, J. 1996. La utilización de la fauna silvestre en la América Tropical y recomendaciones para su manejo sustentable en las sabanas. Ecotropicos 9(2):71-82.

POLANCO, R. 2000. Diagnóstico del uso y comercio de fauna silvestre en el Caribe, Pacífico, Andes, Amazonia y Orinoquia colombiana. Instituto de Investigación en Recursos Biológicos Alexander von Humboldt. Bogotá, Colombia.

PRITCHARD, C.P.; TREBBAU, P. 1984. The turtles of Venezuela. Society for the Study of Amphibians and Reptiles. Venezuela.

RAMÍREZ-PERILLA, J. 1996. Tradición de uso y aprovechamiento de fauna silvestre: límites de la sostenibilidad y acciones posibles. Fundación Natura, Organización Regional Indígena Emberá Wounaan-Orewa-Organización de Estados Iberoamericanos para la Educación la Ciencia y la Cultura, Unidad Administrativa Especial del Sistema de Parques Naturales Nacionales del Ministerio del Medio Ambiente. Impreandes Presencia S.A. Bogotá, Colombia.

REBELO, G.; PEZZUTI, J.B.C. 2000. Percepções sobre o consumo de quelônios na Amazônia. Sustentabilidade e alternativas ao manejo atual. Ambiente \& Sociedade 6/7:85-104.

REDFORD, K.H.; ROBINSON, J.G. 1991. Subsistence and commercial uses of wildlife in Latin America.Págs. 6-23. En: Robinson, J.G.; Redford, K.H. (Eds.). Neotropical wildlife use and conservation. The University of Chicago Press, Chicago, USA.

RENGIFO, J.M.; LUNDBERG, M. 1999. Anfibios y Reptiles de Urrá. Guía de Campo. Skanka. Editorial Colina S.A. Medellín, Colombia. 
ROBINSON, J.G.; BENNETT, E.L. 2000. Carrying Capacity Limits to Sustainable Hunting. Págs. 13-30. En: Robinson, J.G.; Bennett, E.L. (Eds.). Sustainability in Tropical Forests. Columbia University Press, N.Y, USA.

RODRÍGUEZ, J.P.; ROJAS-SUAREZ, F. 1995. Libro Rojo de la fauna Venezolana. Provita. Caracas, Venezuela.

STEARMAN, A.M. 2000. A Pound of Flesh: Social Change and Modernization as Factors in Hunting Sustainability Among Neotropical Indigenous Societies. Págs. 233-250. En: Robinson, J.G.; Bennett, E.L. (Eds.). Sustainability in Tropical Forests. Columbia University Press, N.Y, USA.

TORRES, B. 1999.Plantas, curanderos y prospección biológica. Ciencias 5556: $54-60$.

VICKERS, WT.1984. The faunal components of lowland South American hunting kills. Interciencia 9:366-376. 